Journal Club

Editor's Note: These short, critical reviews of recent papers in the Journal, written exclusively by graduate students or postdoctoral fellows, are intended to summarize the important findings of the paper and provide additional insight and commentary. For more information on the format and purpose of the Journal Club, please see http://www.jneurosci.org/misc/ifa_features.shtml.

\title{
Capitalizing on Directed Evolution and Rational Protein Engineering to Expand the Neuroscientist's Imaging Toolbox
}

\author{
Kevin F. H. Lee and Cary Soares \\ Department of Cellular and Molecular Medicine, University of Ottawa, Ottawa, Ontario, K1G8M5, Canada \\ Review of Abdelfattah et al.
}

Breakthroughs in neuroscience research are accelerated by technological innovations. For example, patch-clamp electrophysiology significantly transformed the brain research landscape in the late 20th century. Neuroscience has now entered into an "age of light” (Scanziani and Hausser, 2009), in which innovations in fluorescence imaging and molecular genetics have provided novel tools for visualizing and manipulating neuronal activity, thus enabling researchers to explore previously inconceivable research questions. These innovations include the discovery and refinement of optogenetic actuators, such as channelrhodopsin and halorhodopsin (Deisseroth, 2015), as well as the development of novel fluorescent protein (FP)-based sensors. Although organic dyes offer sound alternatives to proteinbased sensors for both calcium and voltage imaging (Homma et al., 2009; Ross et al., 2015), a major impetus for developing genetically encoded sensors is the advantage of expressing these tools in a cell-type-specific manner. Additionally, there is significant interest in developing fluorescent probes with

Received March 10, 2016; revised April 4, 2016; accepted April 8, 2016.

Dr Jean-Claude Béique is funded by the Natural Sciences and Engineering Research Council of Canada, the Canadian Institutes of Health Research, the Heart and Stroke Foundation Canadian Partnership for Stroke Recovery, and the University of Ottawa Brain and Mind Research Institute. We thank our mentor Dr Jean-Claude Béique and members of the laboratory for making research a fun and thoroughly rewarding endeavor.

The authors declare no competing financial interests.

Correspondence should be addressed to Kevin F. H. Lee, 451 Smyth

Road, RGN 3501, Ottawa, Ontario, K1G8M5, Canada. E-mail: kevin.fh.lee@gmail.com.

DOI:10.1523/JNEUROSCI.0793-16.2016

Copyright $\odot 2016$ the authors $\quad 0270-6474 / 16 / 365431-03 \$ 15.00 / 0$ red-shifted excitation and emission spectra to add to existing blue/green probes. Redshifted probes not only facilitate imaging deeper in tissue and reduce the risks of phototoxicity, but they also allow for the use of two (or possibly more) spectrally distinct reporters and actuators for all-optical interrogation of brain function, which is likely to be less invasive and more specific than electrophysiological recordings. Despite significant progress in the refinement and spectral expansion of genetically encoded calcium indicators (GECIs; Knopfel, 2012), efforts to improve the performance and utility of genetically encoded voltageindicators (GEVIs) have lagged.

In a recent article in The Journal of Neuroscience, Abdelfattah et al., (2016) provided an excellent demonstration of protein engineering to expand the existing palette of GEVIs. Using rational design strategies and multiple rounds of directed evolution, the authors developed a bright fluorescent GEVI that has a red-shifted excitation and emission spectrum called "FlicR1." The workflow for generating this new voltage sensor involved the same fundamental techniques that led to the development of monomeric red fluorescent protein (mRFP) from DsRed (Campbell et al., 2002), and the expansion of mRFP into widely used variants, such as mCherry (Shaner et al., 2004); tools that are now virtually ubiquitous and indispensable in laboratories that perform livecell fluorescence imaging. In principle, the process of directed evolution can be gen- eralized to refine the functional properties of any protein of interest.

In broad terms, directed evolution involves multiple rounds of random mutagenesis and targeted selection to iteratively refine desired properties of a given protein. To start, one must build a library of gene variants encoding the protein of interest. Abdelfattah et al., (2016) used a rational design approach to begin the process of developing FlicR1 by generating a chimera between a circularly permuted red-shifted FP, "cpmApple," and the voltage-sensitive domain (VSD) from Ciona intestinalis, "CiVSD" (Abdelfattah et al., 2016, their Fig. $1 A)$. This design was chosen because CiVSD has been shown to be localized to membranes in mammalian cells, and because conformational changes induced by the voltage-dependent movement of CiVSD were predicted to efficiently couple to changes in the chromophore environment of circularly permuted FPs, such as cpmApple.

Directed evolution was then conducted through multiple cycles of a high-throughput workflow involving two stages (Abdelfattah et al., 2016, their Fig. 1C). In the first stage of each cycle, a library of variants of the CiVSD-cpmApple chimera was transformed into Escherichia coli bacterial colonies and screened for brightness on a custom wide-field imaging apparatus, allowing the brightest variants to be selected and cultured for further evaluation. In the second stage, the selected constructs were transfected into HeLa cells to screen for proper membrane localization and to assess 
voltage sensitivity via whole-cell electrophysiology. The variants that exhibited the best voltage-sensitivity were chosen for the next cycle of evolution, in which the entire process was repeated. To quantify improvements in voltage sensitivity through successive cycles of evolution, FlicR variants were cotransfected with the green GEVI ArcLight Q239 to serve as a reference standard. To begin each successive round of evolution, new FlicR variants with novel mutations were generated via error-prone DNA replication using the variants selected from the previous round as templates.

When random mutagenesis gives rise to functional enhancements of a protein, researchers can step in to rationally direct further fine-tuning. Abdelfattah et al., (2016) demonstrated this in the final stages of FlicR evolution to specifically improve voltage sensitivity. First, they identified a valine-toalanine mutation at position 207 (on the S3-S4 linker to CiVSD) that was responsible for a marked improvement in FlicR's voltage sensitivity compared to previous versions. Residue 207 was then mutated to all 20 possible amino acids, which led the authors to zero-in on a valine-to-phenylalanine mutation that conferred the greatest improvement to voltage sensitivity. Through a total of eight rounds of bacterial transformations, brightness screenings, voltage testing and clone selection, Abdelfattah et al., (2016) developed the final variant, FlicR1, which had 12 mutations and 16-fold greater brightness compared to its first generation, as well as $>3$-fold larger voltage-dependent fluorescence changes and significantly faster on/off kinetics compared with ArcLight Q239.

As noted by Abdelfattah et al., (2016), the development of GEVIs has trailed behind that of GECIs. Although calcium indicators in general have contributed to neuroscience discoveries at many levels, from single synapses and dendrites (Sabatini et al., 2002; Lee et al., 2016) to neuronal population dynamics in vivo (Dombeck et al., 2007; Tian et al., 2009), researchers stand to benefit considerably from the refinement of voltage sensors given the intrinsic limitations of calcium indicators. First, calcium indicators are generally limited to reporting suprathreshold spiking activity, and exhibit relatively slow kinetics that preclude their ability to report fast spiking or burst dynamics. Also, at the level of the synapse, calcium signal detection typically requires large membrane depolarizations or less physiological low-magnesium recording conditions. Finally, calcium is an important signaling molecule, and although buffer capacity of a given calcium indicator can be estimated, it is not clear how the presence of these mobile calcium-binding molecules alter calcium-dependent processes and homeostasis when expressed in cells for prolonged periods of time. Voltage sensors, such as FlicR1 and ASAP1, are thus poised to overcome some of these major limitations. For instance, the fast kinetics of FlicR1 and ASAP1 enable the resolution of single action potentials up to $100 \mathrm{~Hz}$ (Abdelfattah et al., 2016, their Fig. 5F); something that is currently impossible to accomplish with calcium indicators. Additionally, voltage sensors are in principle less likely to disrupt calcium-dependent physiological processes. Unfortunately, it remains difficult to discriminate small voltage changes with FlicR1 and other available voltage sensors $(\sim 0.066 \% \Delta \mathrm{F} / \mathrm{F}$ per $1 \mathrm{mV}$ change for FlicR1), which largely precludes their use for studying synaptic function and plasticity at dendritic spines. Nevertheless, the work of Abdelfattah et al., (2016) leads in a promising direction, as continued improvements to fast FP-based voltage sensors, such as FlicR1 and ASAP1, will likely bring researchers closer to monitoring both subthreshold and suprathreshold neuronal activity with sensitivity and temporal resolution that increasingly approach electrophysiological standards.

One important limitation of FlicR1 is that it exhibited significant photobleaching, a disadvantage shared among GEVIs in general. Photobleaching of FlicR1 was pronounced using both continuous-wave $(561 \mathrm{~nm})$ and two-photon (1120 nm) laser excitation, reducing FlicR1 fluorescence by more than one-half in $<2$ min of imaging (Abdelfattah et al., 2016, their Figs. 3I, 4C). Although fluorescence signals can be corrected for photobleaching post hoc (as the authors did), and imaging parameters may be adjusted to partially mitigate fluorescence loss, it is not clear whether and how FlicR1 photobleaching affects the voltage readout accuracy over time, and whether this might prohibit long-duration imaging studies lasting tens of minutes or even hours-time scales that are particularly interesting for plasticity studies.

Questions of photostability aside, FlicR1 exhibits voltage responses with superior response amplitudes and faster kinetics compared to existing red-shifted FP-based GEVIs (Perron et al., 2009), joining a rapidly growing palette of red-shifted optogenetic tools including calcium indicators, such as R-GECO (Zhao et al., 2011) and R-CaMP (Akerboom et al., 2013), and channelrhodopsin-like actuators, such as ReaChR (Lin et al., 2013), VChR1 (Zhang et al., 2008), and ChRimson (Klapoetke et al., 2014). As noted above, perhaps the most interesting upside to developing tools with red-shifted spectra is the potential for using multiple spectrally separated reporters and actuators in combination. Abdelfattah et al., (2016) explored this possibility in "all optical electrophysiology" experiments, pairing FlicR1 with channelrhodopsin variants in both HeLa cells and cultured hippocampal neurons to visualize light-evoked changes in membrane potential (Abdelfattah et al., 2016, their Figs. 7, 8). Although these experiments were demonstrated to be feasible, the FlicR1 chromophore was found to be excited by the blue light used to activate the channelrhodopsins in neurons, necessitating adjustments to the illumination strategy to minimize this effect. Additionally, it was found that the $561 \mathrm{~nm}$ laser used to excite FlicR1 could also directly activate channelrhodopsin and depolarize neurons by as much as $14 \mathrm{mV}$. Therefore, any attempts to use all-optical strategies for neurophysiology using presently available tools will require careful experimental design involving thoughtful selection of optical sensors to mitigate spectral crosstalk and, undoubtedly, rigorous electrophysiological validation.

Over the past two decades or so, imaging techniques have taken a prominent role in modern neuroscience laboratories around the world, and the continued development of optical tools will be integral for expanding the kinds of questions researchers can explore. The refinement of genetically encoded proteins through directed evolution and rational protein engineering, as demonstrated Abdelfattah et al., (2016) will improve our ability to peer into and manipulate the functions of brain circuits to help answer enigmatic questions of learning and memory, emotions, behavior, and consciousness for decades to come.

\section{References}

Abdelfattah AS, Farhi SL, Zhao Y, Brinks D, Zou P, Ruangkittisakul A, Platisa J, Pieribone VA, Ballanyi K, Cohen AE, Campbell RE (2016) A bright and fast red fluorescent protein voltage indicator that reports neuronal activity in organotypic brain slices. J Neurosci 36:24582472. CrossRef Medline

Akerboom J, Carreras Calderón N, Tian L, Wabnig S, Prigge M, Tolö J, Gordus A, Orger MB, Severi KE, Macklin JJ, Patel R, Pulver SR, Wardill TJ, Fischer E, Schüler C, Chen TW, Sarkisyan KS, Marvin JS, Bargmann CI, Kim DS, et al. (2013) Genetically encoded calcium indicators for multi-color neural activity imaging and combination with optogenetics. Front Mol Neurosci 6:2. CrossRef Medline

Campbell RE, Tour O, Palmer AE, Steinbach PA, 
Baird GS, Zacharias DA, Tsien RY (2002) A monomeric red fluorescent protein. Proc Natl Acad Sci U S A 99:7877-7882. CrossRef

Deisseroth K (2015) Optogenetics: 10 years of microbial opsins in neuroscience. Nat Neurosci 18:1213-1225. CrossRef Medline

Dombeck DA, Khabbaz AN, Collman F, Adelman TL, Tank DW (2007) Imaging large-scale neural activity with cellular resolution in awake, mobile mice. Neuron 56:43-57. CrossRef Medline

Homma R, Baker BJ, Jin L, Garaschuk O, Konnerth A, Cohen LB, Zecevic D (2009) Widefield and two-photon imaging of brain activity with voltage- and calcium-sensitive dyes. Philos Trans R Soc Lond B Biol Sci 364:24532467. CrossRef Medline

Klapoetke NC, Murata Y, Kim SS, Pulver SR, Birdsey-Benson A, Cho YK, Morimoto TK, Chuong AS, Carpenter EJ, Tian Z, Wang J, Xie Y, Yan Z, Zhang Y, Chow BY, Surek B, Melkonian $\mathrm{M}$, Jayaraman $\mathrm{V}$, Constantine-Paton $\mathrm{M}$, Wong GK, et al. (2014) Independent optical excitation of distinct neural populations. Nat Methods 11:338-346. CrossRef Medline

Knopfel T (2012) Genetically encoded optical indicators for the analysis of neuronal circuits.
Nat Rev Neurosci 13:687-700. CrossRef Medline

Lee KF, Soares C, Thivierge JP, Béique JC (2016) Correlated synaptic inputs drive dendritic calcium amplification and cooperative plasticity during clustered synapse development. Neuron 89:784-799. CrossRef Medline

Lin JY, Knutsen PM, Muller A, Kleinfeld D, Tsien RY (2013) ReaChR: a red-shifted variant of channelrhodopsin enables deep transcranial optogenetic excitation. Nat Neurosci 16: 1499-1508. CrossRef Medline

Perron A, Mutoh H, Launey T, Knöpfel T (2009) Red-shifted voltage-sensitive fluorescent proteins. Chem Biol 16:1268-1277. CrossRef Medline

Ross WN, Miyazaki K, Popovic MA, Zecevic D (2015) Imaging with organic indicators and high-speed charge-coupled device cameras in neurons: some applications where these classic techniques have advantages. Neurophotonics 2:021005. CrossRef Medline

Sabatini BL, Oertner TG, Svoboda K (2002) The life cycle of $\mathrm{Ca}(2+)$ ions in dendritic spines. Neuron 33:439-452. CrossRef Medline

Scanziani M, Hausser M (2009) Electrophysiology in the age of light. Nature 461:930-939. CrossRef Medline
Shaner NC, Campbell RE, Steinbach PA, Giepmans BN, Palmer AE, Tsien RY (2004) Improved monomeric red, orange and yellow fluorescent proteins derived from Discosoma sp. red fluorescent protein. Nat Biotechnol 22: 1567-1572. CrossRef Medline

Tian L, Hires SA, Mao T, Huber D, Chiappe ME, Chalasani SH, Petreanu L, Akerboom J, McKinney SA, Schreiter ER, Bargmann CI, Jayaraman V, Svoboda K, Looger LL (2009) Imaging neural activity in worms, flies and mice with improved GCaMP calcium indicators. Nat Methods 6:875-881. CrossRef Medline

Zhang F, Prigge M, Beyrière F, Tsunoda SP, Mattis J, Yizhar O, Hegemann P, Deisseroth K (2008) Red-shifted optogenetic excitation: a tool for fast neural control derived from Volvox carteri. Nat Neurosci 11:631-633. CrossRef Medline

Zhao Y, Araki S, Wu J, Teramoto T, Chang YF, Nakano M, Abdelfattah AS, Fujiwara M, Ishihara T, Nagai T, Campbell RE (2011) An expanded palette of genetically encoded $\mathrm{Ca}(2)(+)$ indicators. Science 333:18881891. CrossRef Medline 